\title{
Mapping Geological Structures in Ilbisil Area, Kajiado County Using Remote Sensing Techniques
}

\author{
Dr. Aaron K. Waswa \\ University of Nairobi, Department of Geology, P.O B0X 30197-00100 Nairobi \\ Jullian A. Ogendo \\ University of Nairobi, Department of Geology, P.O B0X 30197-00100 Nairobi
}

\begin{abstract}
This project is all about the applications of remote sensing techniques in mapping the geological structures in Ilbisil area, Kajiado county. Lineament mapping constitute a very important process in the field of Geology and Earth Sciences. Much of the exploration work, mapping and the exploitation of natural resources including oil and gas, ground water and other hydrocarbons rely on a thorough understanding of the geological structures within an area and the ability to interpret the result from analysis before the exploitation process begins. This increases success rates while reducing cost and time associated with geophysical surveys. Understanding structures is the key to interpreting crustal movements that have shaped the present terrain. Geological structures play an important role in mineral and hydrocarbon exploration and potential hazard identification and monitoring. Structures can also indicate potential locations of oil and gas reserves by characterizing both the underlying subsurface geometry of rock units and the amount of crustal deformation and stress experienced in a certain locale. Remote sensing has the advantage of covering a huge area while data about features at different frequencies recorded in different spectral bands which can be exploited to obtain information about the structural geology of the area. The area of study is characterized by dense forest vegetation with bushes and thickets with difficult access, scarcity and discontinuity of the available outcrops. Geological and structural mapping therefore in this type of environment is difficult, time consuming, expensive and even dangerous and sometimes dangerous. All these factors constitute a major problem for the accurate geological mapping in the area.This study attempts to employ the use of modern satellites technology in trying to map the near surface structures and to compare and update the existing structural and geological maps. Satellite imagery have a synoptic view of the earth's surface and therefore it is possible to acquire data in inaccessible and in huge areas. The objective of this study is to map the geological structures in Kajiado area using remote sensing techniques. The expected output is to show how the mapping method integrating remote sensing technology is useful for accurate interpretation of geological structures by designing a geodatabase for structural mapping and also to document a methodology for structural mapping using remote sensing technology. The methods to be used in this study include Pan sharpening and image sub-setting, Principal component analysis, Band ratios and combinations, Spatial filtering, Sobel filtering, Edge enhancement and Geodatabase scheme. The materials include; Landsat 8, DEM/ SRTM, Topographical and geological maps and Satellite imagery.
\end{abstract}

Keywords: Geological structure, Remote sensing

DOI: $10.7176 /$ JEES/9-11-05

Publication date: November $30^{\text {th }} 2019$

\subsection{MATERIALS AND METHODS}

1.10 Study Area

Ilbisil occurs within the Neoproterozoic Mozambique Belt in Kenya located in Ilbisil area, central Kajiado sub county, Kajiado county. The study area is bounded by longitudes 9752000 to 9780000 and latitudes 23200 to 27400. (2015 Kenya information guide. Com) [1]

The choice of this area and its extent was informed by previous geological mapping exercise carried out by Saggerson in the year 1957 which mapped the entire topography of the area mainly focusing on the geology and structural features present and visible during that time. (Saggerson. (1957). [2] 

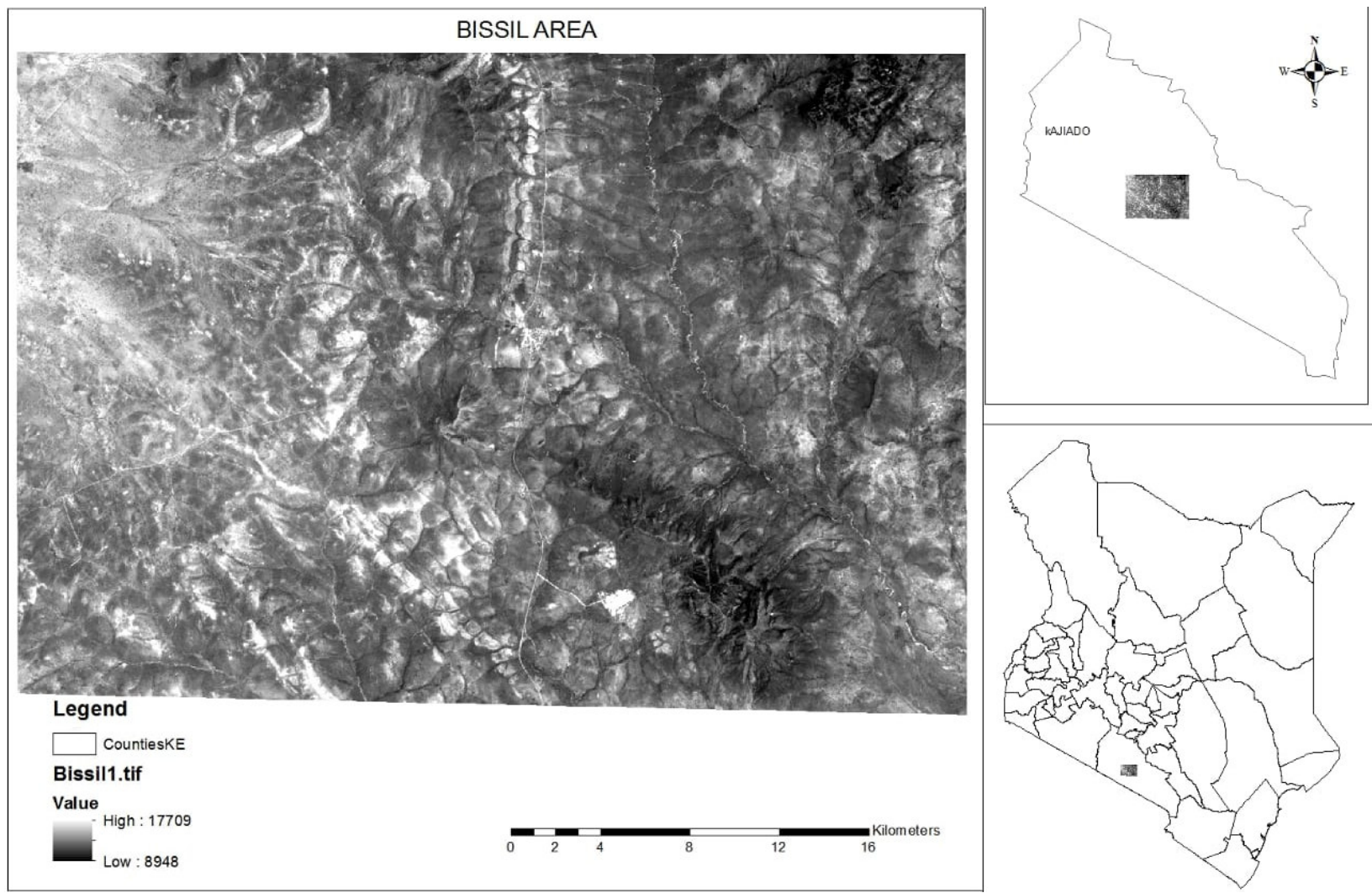

Figure 1 of a map showing the location of Ilbisil area in Kenya

\subsection{Geological setting}

The rocks of Ilbissil area fall into the categories of: Archean rocks of the Basement System; Tertiary Yatta Plateau system and the superficial deposits of Pleistocene to Recent age.

The Basement System rocks are mainly metamorphosed sediments now altered to marbles, gneisses and granulites. They form part of an extensive belt of similar Archaean rocks which have an approximate north-west to south-east trend from Maralal to the south Kenya border, and through Tanganyika to Mozambique (Saggerson, 1957). [2]

Rocks of the Basement System, form the oldest rocks and are entirely of sedimentary origin. Minor igneous activity following the deposition of the sediments is represented by amphibolites which are thin and concordant.

Structures of the Basement System were then produced by orogenic folding. NNW-SSE minor folds are abundant along the river Kajiado so their major folds are probably aligned in the same direction.

Climate of the area is predominantly semi-arid. The annual temperature is about $18.9{ }^{\circ} \mathrm{C}$. The area receives approximately $500 \mathrm{~mm}$ of rainfall annually. Precipitation pattern in the area is bimodal, with long rains recorded in the months of March to May and the short rains from October to December. (Kajiado county government website) [3]

The area is dominated by open grass plains, acacia woodlands, rocky thorn bushlands, swamps, marshland and scarce vegetation in low lying areas which increases with altitude.

\subsection{Data}

The Landsat 8 was used in this study and it is the latest in a series of Landsat space mission which began operation on May 30, 2013. On board, are two instruments: the operational Land Imager (OLI) which includes refined heritage bands, along with three new bands namely: a deep blue band for coastal/aerosol studies, a shortwave infrared band for cirrus detection and a quality assessment band (USGS, 2019) [4]. Landsat have proven useful in mapping both geology and the structure because of their ability to enhance spectral lithological contrast thus helping in discriminating lithological units (Ilhan.K.V. (1978).) [5] This is because of their ability in mapping and exploration of geological resources like water, oil and hydrocarbons.

The main source of data for structural analysis and mapping in this study is the Landsat 8 captured on $21^{\text {st }}$ January 2017. 


\subsection{METHODOLOGY}

\subsection{Acquiring Landsat Data}

The required images are selected from the mono-log window of the web-based application Earth explorer that enables one to select the images needed based on the location by providing either the name or geolocation of the study area. Window therefore allows the selection of different types of Landsat images with clear definition of the date. The images were then submitted to the USGS servers for correction and it was later on downloaded through the services known as Bulk Download that is made available by the USGS website which enables one to choose the images for the study, submit for correction and then download the images once they are ready. (USGS. 2019). [6]

\subsection{Pan Sharpening and Image sub-setting}

Using the panchromatic band 8 of the Landsat ETM+ with a spatial resolution of 15 meters, bands 1-5 and band 7 were pan sharpened using the resolution merge algorithm. The principal component method was applied together with the cubic convolution resampling method resulting in a smoother image with a resolution of 15 meters.

Sub-setting was then performed on all the eight bands of the ETM+. The output extent was defined by a subset of the study area coverage applied to mask other bands too. Before sub-setting, the original image had a width of $8,586 \mathrm{~m}$ and a height of 7,617 with a standard deviation of 11.14 .

The output image had a width of $1942 \times 1930$ height with a standard deviation of 5.49. The pixel size in both images in both $\mathrm{x}$ and $\mathrm{y}$ remained as 28.50 meters. The implication of the sub-setting was seen during data analysis and processing by reducing the time taken during processing. The spatial sub-setting was also separately performed on bands 3, 4, 5 and 7 of the Landsat ETM+ and the ratio of bands 3 and 4 .

Enhancement and filtering were also done separately in Erdas Imagine $C 2013$ software for bands 5 and 7 and also a combination of bands $4+5+7$ from the original subset image of Landsat ETM+ image.

The 30m DEM from Aster representing the study area was extracted in ArcGISC 10.2 software. The result was a DEM covering the four corners of the study area extent. This DEM was then used to extract a shaded relief of the study area using the 3D Analysis extension in ArcGIS with an azimuth angle of 315 degrees at an altitude of 45 degrees in order to illuminate and enhance the geomorphology. The output of this process was overlaid on all the processed images to help identify and extract structural features in the Landsat images.

\subsection{Principal component analysis (PCA)}

PCA is used to identify data patterns and in data expression in such a way that highlight their intercorrelations. The covariance of each band was computed and the results used to calculate the eigenvectors and eigenvalues. Eigenvectors with the highest eigenvalues formed the principal component of the data set. The eigenvectors give us information about the patterns in our data where a line of best fit is being drawn following the concentration of most values. Values representing lineaments within the image are therefore expected to concentrate and align in a direction corresponding to the structures within that locality. This orientation will guide in the process of visual interpretation and the extraction of linear features in the image.

Using the false color IR RGB $=643$ and the cubic convolution resampling, the final image will be interpreted for linear structures.

\subsection{Band ratios and combinations}

A combination of bands $4+5+7$ and the false color composite images of bands RGB $=321$ was helpful in the interpretation of geological structures by distinguishing structures from the land surface in the area of study like roads. The false color composite represented by bands $\mathrm{RGB}=432$ combination was helpful in mapping the vegetation covers in the study area.

\subsection{Edge enhancement}

Edge enhancement technique basically strengthens the local contrast according to (Olgen, 2004). [7] The algorithm is based on a window which is moved systematically through the image and centered on each pixel. An average value calculated at each position is compared to the averages of the neighboring pixels. (Kumar, 2014). [8] Using the linear stretching method, with a slope of 20 and a shift of 20, other non-structural features were masked out leaving mainly linear features with higher brightness values giving a clearer view of the structural trend in the area.

\subsection{RESULTS}

\subsection{Lineaments Extraction}

Lineaments occur as straight, curvilinear, parallel or en-echelon features. They are related to fracture systems, discontinuity planes and shear zones in rocks. Dykes and veins also appear as lineaments. The term also includes fracture traces described from aerial photographic interpretation. (Gupta, 2003). [9] 
The extraction of lineaments from Landsat 8 panchromatic band of the area of study was done under default parameters of the Arc Gis software. These lineaments have been visually edited to extract only the structural lineaments using an image from band 3. (Kassou, A., Essahlaoui, A., \& Aissa, M. (2012).) [10]

The rose diagram of these lineaments shows that their predominant trend is in NW-SE direction. Most of the structures were trending towards NW-SE following the general direction of structures forming the Mozambique belt system.

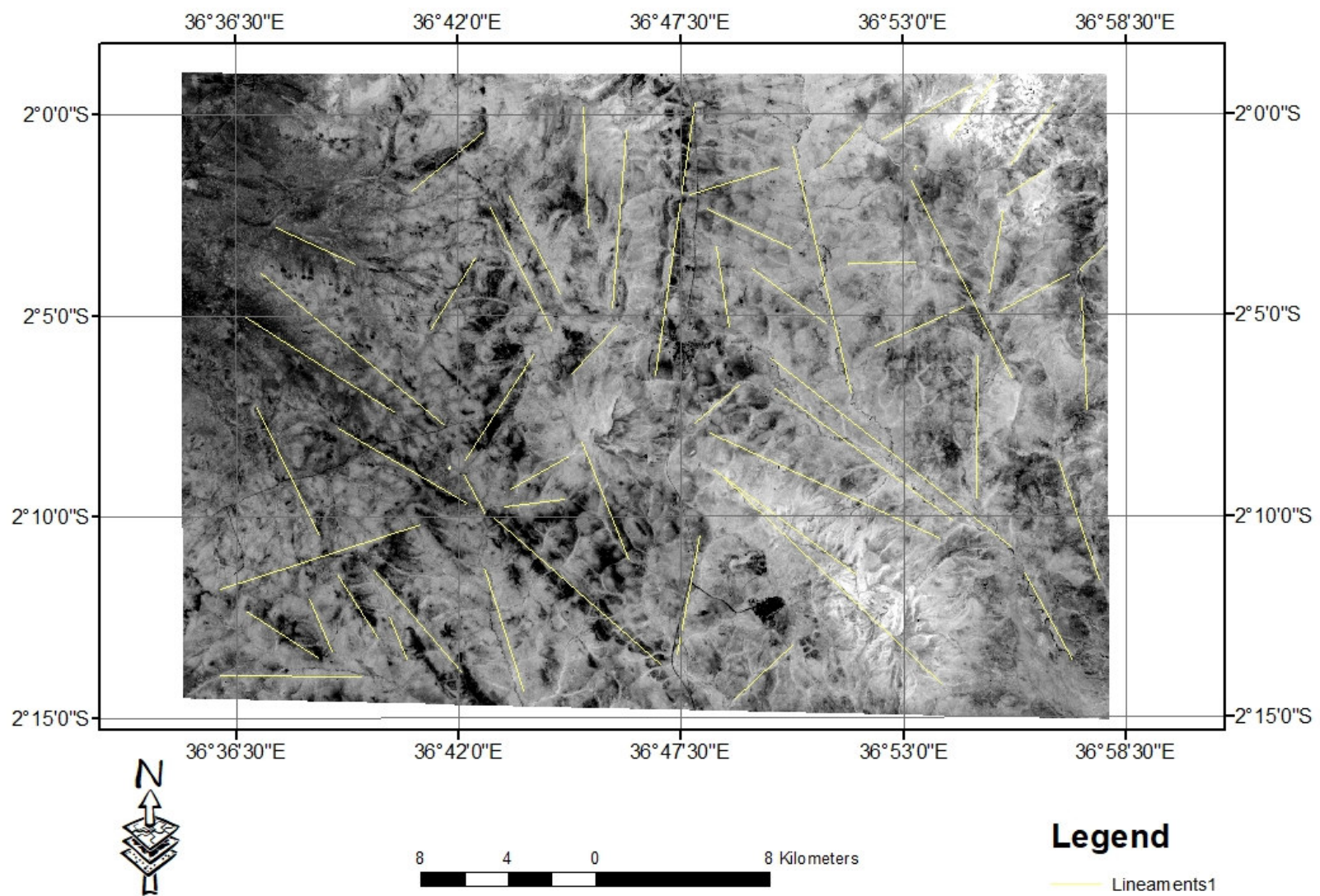

Figure 2 of a map showing distribution of lineaments

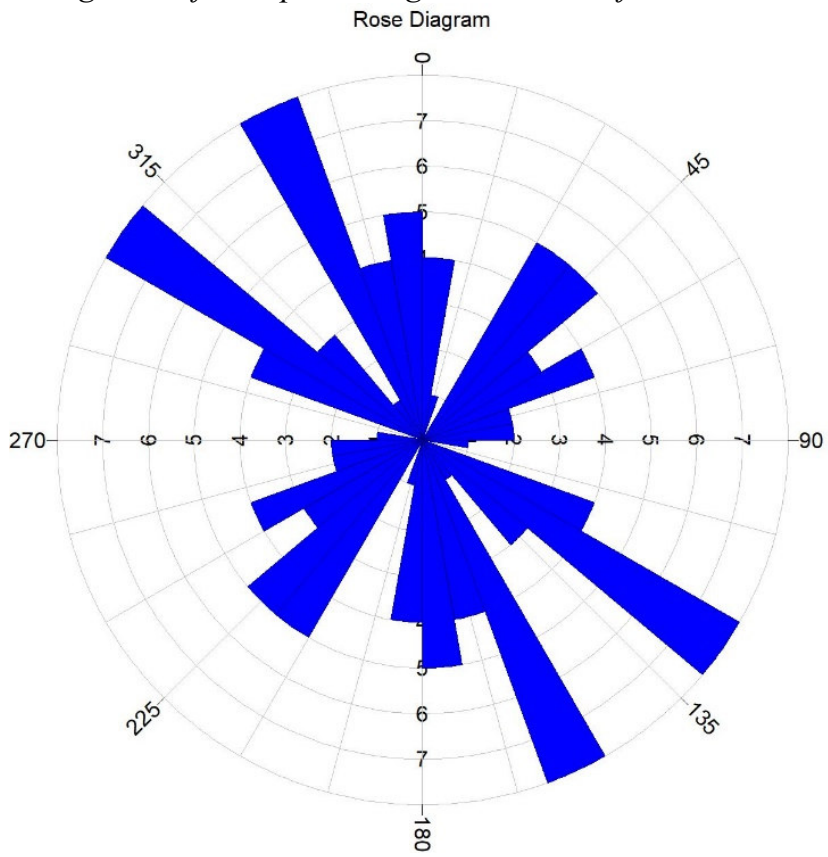

Figure 3 of a Rose Diagram for lineaments drawn to show the general structural orientation and trend 


\subsection{Band Ratio and Combination}

Because different features have different spectral signatures, color has been used to differentiate different objects on the surface of the earth like water, soil and vegetation. In order to achieve this, different band combinations are used to enhance certain features while suppressing others in order to extract information. The false color images consisting of bands 432 and 743 have been used to interpret geological structures. The use of color also helps in depicting an object's outline thus giving us an idea of its extent and shape. The texture of an object and the shadows can also be observed using color. (Demirkesen, 2014). [11]

The false color composite of bands $\mathrm{RGB}=743$ is always used for vegetation mapping, monitoring drainage and soil patterns. The green hues represent a healthier vegetation while lighter shades of green indicate sparsely vegetated areas.

From the interpretation of the results, it was observed that vegetation distribution represented by the green color in the false color image, mainly concentrated and followed the direction of the lineaments. Vegetation in false color image, can be seen to follow a North/West-South/East trend following the folds. Much of the structural concentration is seen in the eastern part. Areas concentrated by structures also appear in shades of purple color from the composite image where the red, green and blue channels are represented by bands 7,4 and 3 respectively.

\section{Band Ratio and Combination}

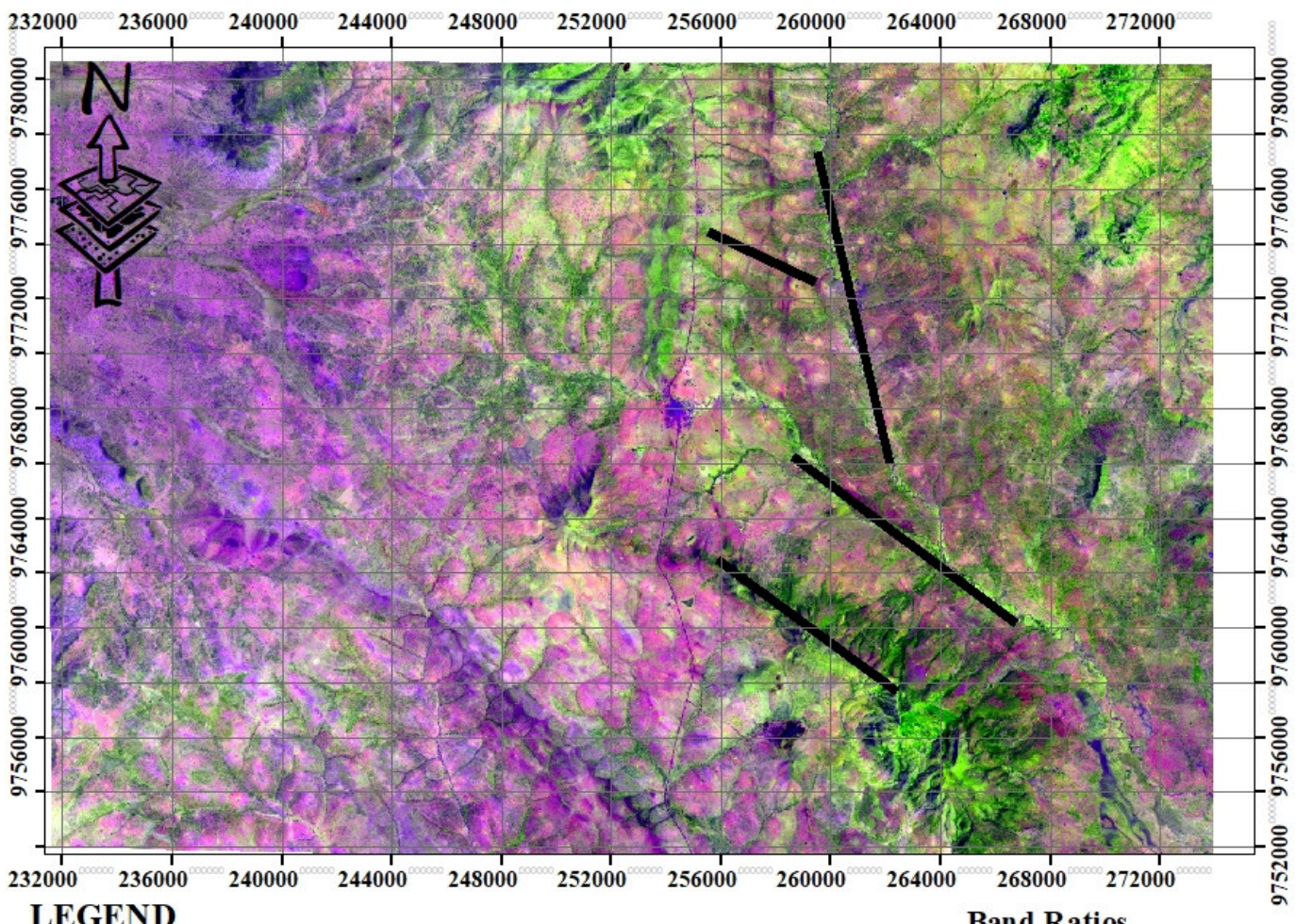

LEGEND

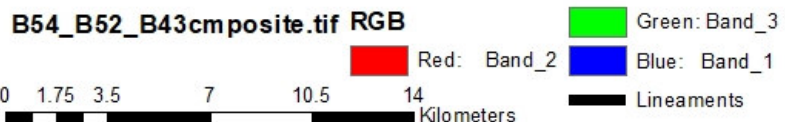

Band Ratios

\section{B5/B4 B5/B2 B4/B3}

Author: OGENDO JULLIAN ADHIAMBO

Figure 4 showing a False color composite image Band 743

\subsection{Principal Component Analysis}

Principal component analysis is a way of identifying patterns in data, and expressing the data in such a way as to highlight their similarities and differences. (Randranasalo, E.B (2009)) [13]

The PCA done on the 8 bands of the Landsat ETM+ was mainly aimed at reducing redundancies in the correlated bands resulting to a much sharper and clearer image for structural interpretation.

Surface features are captured and are represented by the reddish color. The RGB 742 image helps to identify folds; the shadow effect helps to produce a 3-D look and also displays the general slope direction. Color also helped in the interpretation and the identification of lineaments. The lineaments tended to have the greenish color. The tonal differences from principal component image showed very small details including minor structural features that were not discrete in the original image. The main drainage channels were also captured in blue color. Their flow- direction follows the general trending direction of the lineaments which indicate an association and also justify the influence of structures on the flow of the rivers. 


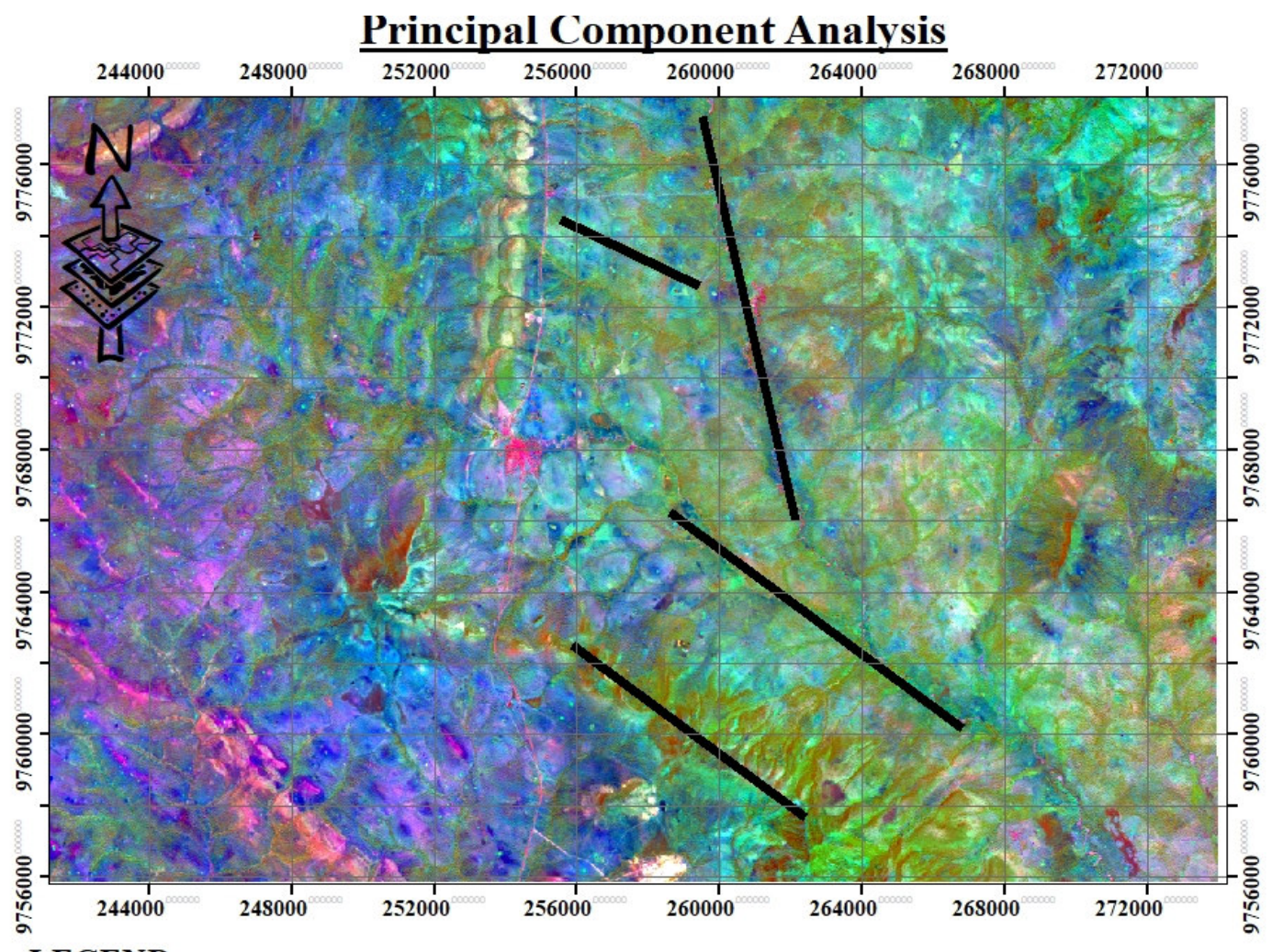

LEGEND

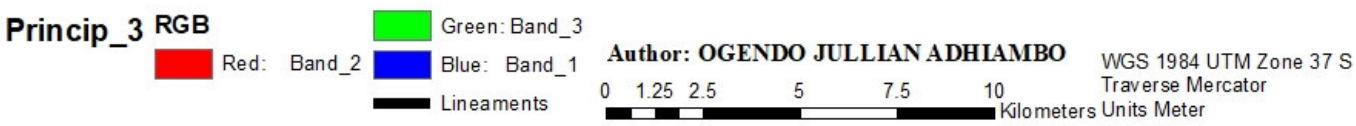

Figure 5 showing $P C A R G B=742$

\subsection{Left Diagonal Edge Detect - Band 4}

Various lineations can be seen on the image after performing a $3 \times 3$ Left diagonal edge enhancement detection filter and a linear enhancement by adjusting the slope to a value of 3.10 with a shift of 20 . The shift aided in enhancing lineation by increasing their brightness values of the structures.

\subsection{Left Diagonal Edge Detect - Band 3and 4 ratio}

Band 3 in the ratio between bands 3 and 4 of the ETM+ image helped in reducing brightness values in the pixels which were predominant in band 4 . The surfaces with pixels depicting higher values were observed on bare rocky surfaces and river beds which are majorly filled with sediments showing much of the electromagnetic energy hitting the surface yielding in a continuous smooth surface. The output of the ratio yielded an image with lesser brighter values while the surrounding pixels retained the brightness, which helped in accentuating the structures.

\subsection{DISCUSSION}

This study presents the potential of Landsat imager specifically the Landsat 8 image in mapping lineaments in Kajiado Central, Kajiado County. In the first stages, the Landsat 8 image had to undergo some pre-processing before the extraction process began. To reduce the processing time, a subset of bands 4,7 , the ratio of bands 3 and 4 and a composite image were made using a predefined boundary marking the extent of the area of study. A principle component analysis was applied on bands 1 to 5 which have a spatial resolution of 15 meters after resampling in order to generate an uncorrelated image that would show patterns in the structural orientation. The false color bands combination of bands $\mathrm{RGB}=742$ helped in validating the orientation of the lineaments. The shadow that resulted from PCA helped in emphasizing structures making it easy to identify the morphology and the tectonic processes that led to the formation of these lineaments. The result obtained by combining bands 432 false color composite revealed a relationship between vegetation distribution, their density and pattern in the area with geological structures. It was evident that the orientation followed a linear pattern indicating an influence of structures on vegetation distribution.

The approach applied in the extraction of lineaments for this study include: the directional filters consisting of right and the left diagonal edge filters. The right and left diagonal edge detect filters were specifically applied 
to detect lineaments with either right or left orientation. According to the result observed from the analysis, right diagonal filter applied on band 7 was only able to identify a few structures which were mostly folds. There was quite a number of lineaments detected using the left diagonal edge detect filter largely because the initial study indicated a North-West/South-East orientation. Band 4 yielded better results compared to band 7. This is because of the interference of neighboring pixel with more or less similar radiance like those in band 7 hence making it difficult to differentiate lineaments from other non-geological features. The results from the ratio of bands 3 and 4 using the left diagonal edge detect filter produced a series of short lineaments that were observed because of the difference in intensity among the neighboring pixels as a result of that ratio.

\subsection{CONCLUSIONS AND RECOMMENDATIONS}

\subsection{Conclusions}

Satellite remote sensing is one of the best techniques for mapping geological structures. This is because of its ability to capture data at different bands of the electromagnetic spectrum using the reflectance energy from objects on the ground and differentiate such objects on the basis of their unique spectral signature. The automation process in feature extraction can be achieved using a variety of image analysis software available. The major challenge lies at the interpretation stage-which remains subjective because it requires human intervention. Different people could interpret or misinterpret different features differently based on their general knowledge of an area or simply by using knowledge acquired through existing literature. Ground-truthing therefore becomes quite critical in order to identify and map the correct features as they appear on a processed satellite image.

Filtering techniques as used in the process of linear feature extraction, is one of the best strategies for mapping lineations and structures because of their ability to establish spectral patterns of the different features and thus clustering similar features together. Different filters yield different results depending on the expected results. Some filters emphasize certain aspects of a feature while de-emphasizing others in an attempt to bring out differences and similarities inherent in the image. Among the structures mapped in this study, folds were the most prominent due to their visible nature on the surface of the earth. The faults present in the area of study were deduced from the interpretation of the tectonic processes visible in the area. The dip seated faults however couldn't be mapped because of the sediments that have covered them over time or the presence of vegetation that obscure their visibility.

Resampling the composite Landsat image of the area of study from the original 30 meters spatial resolution to a finer 15 meters' resolution helped in refining the clarity of the image and hence subtle features which were quite invisible in the original image. Performing a principal component analysis on the 8 bands of the Landsat ETM+ image helped in limiting the redundancies in the data by reducing variability in the bands. To perform this analysis, different band combinations and a ratio were used. A combination of bands 432 were used specifically to map out vegetation in the area and relate their distribution to structures. Areas with vegetation were represented with shades of red, pink-red and dark red depending on the nature and type of vegetation. Based on the results obtained, we therefore can conclude that there is a relationship between structures and vegetation distribution. The drainage pattern appeared to follow the same orientation and pattern similar to structures. We can therefore deduce a direct influence resulting from these structures. Bands 7, 4 and the ratio between bands 3 and 4 were used for extracting lineaments. To achieve this, filtering techniques in Erdas Imagine ${ }^{\circledR} 2013$ and ArcGis 10.5 were used and the result refined further using image enhancement techniques including linear and histogram equalizer.

Of all the bands used for this study, the panchromatic band 4 of the Landsat ETM+ image proved to be more effective in the process of identifying different structures based on the results obtained.

The Right diagonal and Left diagonal edge detection filters used in the study showed a general NW-SE in that image. Enhancement techniques such as the Gamma, Histogram Equalization and the linear stretching helped in either increasing or decreasing the brightness values of pixels in order to enhance their differences. The $3 \times 3$ Sobel 2 filter methods helped in emphasizing surface visible structures like folds. An existing structural map of the area was later used to compare both the orientation and the type of structures mapped.

The use of Landsat ETM+ image therefore has proved that it is possible to map the surface and near-surface geological structures at considerable degree of accuracy and therefore helped in achieving the objectives set out in this study. The methodology used in the study can also be replicated in other studies to map out lineaments.

\subsection{Recommendation}

Most structures are a factor of the geology of an area and therefore, a high-resolution imagery like Aster with 14 bands would be adequate to map small differences in geological units because of the wider spectrum the bands cover. After establishing the geology, it is possible to identify rock units susceptible to earth processes related to folding and faulting because of their physical and chemical properties. Mapping of structures would therefore be focused in this rock units and the deductions made on the type of the structures can be made at an early stage before ground-truthing is carried out. Mapping a considerable huge area of land although maybe costly and timeconsuming undertaking in terms of the volume of data used during the analysis - provides the advantage of observing and establishing the structural trend of an area. Caution should however be taken in line with the 
available resources if other data like magnetic and seismic data are going to be used. This is because of the high costs of acquiring such data.

A combination of high-resolution satellite imagery like SPOT together with Landsat data would improve the result of the mapping process hence making the interpretation of structural features easier. Radar data could also be used due to its longer wavelengths capable of penetrating clouds thereby providing an image free from noise as characterized by Landsat data. The same ability available in radar technology would be instrumental in identifying structures and lineations covered by sediments over time because of erosion and other tectonic processes. Further research should therefore be conducted on the specific bands and band ratios that would yield the best results in structural mapping process. Other data sources like seismic data and magnetic data could be used in combination with remote sensing data so as to not only improve the accuracy of the mapping exercise but also in the process of validating the results obtained. This will help in the process of verifying the authenticity of the structures. It will also be possible to filter out other non-geological linear features from the image like roads and drainage systems which might be misinterpreted as lineations. In conclusion, further research should be conducted in the area to establish whether there's a direct correlation between structural patterns and vegetation distribution and whether such a pattern can be used as a proxy for mapping geological structures.

\section{REFERENCES}

[1]. Kenya information guide. Com., 2015

[2] Saggerson. (1957). Geology of the Ilbisil Area. Nairobi: Government of Kenya Printer.

[3] Kajiado county government website

[4] USGS. (2019,). Landsat 8. Retrieved from Landsat Missions: http://landsat.usgs.gov/landsat8.php

[5] Ilhan.K.V. (1978). Application of LANDSAT imagery to studies of structural geology and geomorphology of the Mentese region southwestern Turkey. Elsevier, 51-60.

[6] USGS. (2019,). USGS Science for a Changing World. Retrieved from Earth Explorer: http://earthexplorer.usgs.gov/

[7] Olgen, K. (2004). Determining Lineaments and Geomorphic features using Landsat 5-TM on the Lower Bakircay plain, western Turkey. Aegean Geographical, 47-57.

[8] Kumar, M. (2019,). WAMIS. Retrieved from World Agro Meteorological Information Service: www.wamis.org/agm/pubs/agm8/Paper-5.pdf

[9] Gupta.P. (2003). Remote Sensing Geology. Berlin: Springer-Verlag Berlin Heidelberg

[10] Kassou, A., Essahlaoui, A., \& Aissa, M. (2012). Extraction of Structural Lineaments from Satellite Images Landsat 7 ETM+ of Tighza Mining District (Central Morocco). Research Journal of Earth Sciences, 44-48.

[11] Demirkesen et al, (2014). International Society for Photogrammetry and Remote Sensing. Retrieved from ISPRS: www.isprs.org/proceedings/xxxv/congress/comm4/papers/503.pdf

[12] Randranasalo, E.B (2009) Structural mapping in the southern Madagascar using Landsat TM data. International Journal of Innovation and Applied Sciences 